\title{
An Updating Method for Finite Element Models of Flexible-Link Mechanisms Based on an Equivalent Rigid-Link System
}

\author{
R. Belotti $\mathbb{D}^{1},{ }^{1}$ R. Caracciolo, ${ }^{2}$ I. Palomba $\mathbb{D}^{1},{ }^{1}$ D. Richiedei $\mathbb{D}^{2},{ }^{2}$ and A. Trevisani $\mathbb{D}^{2}$ \\ ${ }^{1}$ Faculty of Science and Technology, Free University of Bozen-Bolzano, Piazza Università 5, Bolzano, Italy \\ ${ }^{2}$ Dipartimento di Tecnica e Gestione dei sistemi industriali (DTG), Università degli Studi di Padova, Stradella S. Nicola 3 , \\ 36100 Vicenza, Italy \\ Correspondence should be addressed to D. Richiedei; dario.richiedei@unipd.it
}

Received 31 March 2018; Revised 28 June 2018; Accepted 17 July 2018; Published 16 September 2018

Academic Editor: Gianluca Gatti

Copyright $(2018$ R. Belotti et al. This is an open access article distributed under the Creative Commons Attribution License, which permits unrestricted use, distribution, and reproduction in any medium, provided the original work is properly cited.

\begin{abstract}
This paper proposes a comprehensive methodology to update dynamic models of flexible-link mechanisms (FLMs) modeled through ordinary differential equations. The aim is to correct mass, stiffness, and damping matrices of dynamic models, usually based on nominal and uncertain parameters, to accurately represent the main vibrational modes within the bandwidth of interest. Indeed, the availability of accurate models is a fundamental step for the synthesis of effective controllers, state observers, and optimized motion profiles, as those employed in modern control schemes. The method takes advantage of the system dynamic model formulated through finite elements and through the representation of the total motion as the sum of a large rigid-body motion and the elastic deformation. Model updating is not straightforward since the resulting model is nonlinear and its coordinates cannot be directly measured. Hence, the nonlinear model is linearized about an equilibrium point to compute the eigenstructure and to compare it with the results of experimental modal analysis. Once consistency between the model coordinates and the experimental data is obtained through a suitable transformation, model updating has been performed solving a constrained convex optimization problem. Constraints also include results from static tests. Some tools to improve the problem conditioning are also proposed in the formulation adopted, to handle large dimensional models and achieve reliable results. The method has been experimentally applied to a challenging system: a planar six-bar linkage manipulator. The results prove their capability to improve the model accuracy in terms of eigenfrequencies and mode shapes.
\end{abstract}

\section{Introduction}

The use of lightweight mechanisms is increasing significantly in the last years. Indeed, this kind of construction ensures less material and power consumption that allows for smaller actuators. On the other hand, the flexibility of the links makes the motion planning and control critical and hence imposes the use of advanced techniques that account for the flexible system dynamics [1-6]. Therefore, great attention has been paid to dynamic modeling of flexible-link mechanisms (FLMs) to boost the development of effective model-based design or control techniques or to allow for numerical simulations.

Several modeling approaches are proposed in literature. A widespread approach is to take advantage of finite element (FE) methods to discretize the link flexibility through a finite number of elastic degrees of freedom (dofs) and to represent the total motion of the system as the superposition of a large amplitude rigid-body motion and the coupled smallamplitude elastic deformation [5-9].

The availability of a correct model formulation is however not sufficient to predict correctly the dynamic response. Indeed, these FE models are not effective if the inertial and elastic properties of the model are not correctly tuned. Therefore, it is necessary paying attention to the correct estimation of the model parameters, either through direct physical measurement, whenever possible, or using experimental parameter identification techniques. The latter process of correcting the FE model parameters to feature a set of experimental measurements is known in the field of structural dynamics as model updating. 
Model updating can be performed by following two different types of approach: noniterative or iterative techniques $[10,11]$. Recently, semidirect methods have also been proposed [12]. Noniterative techniques (also denoted direct or one-step) provide analytical solutions to model updating problem through a single step [13]. Nonetheless, such solutions might be without physical meaning and scarcely robust to measurement noise $[10,11]$. Conversely, iterative techniques (also denoted parametric techniques) preserve the physical meaning of the updated parameters at the cost of an increase of the computational complexity due to the numerical iterations adopted to reduce the mismatch between experimental and model responses. Basically, iterative techniques compute the updated parameters through an objective function that represents the difference between analytical and experimental results. Therefore, they may lead to local optimal solutions if the updating problem is formulated as a nonconvex optimization problem. In the last years, the use of iterative techniques has been boosted by the advanced techniques and toolboxes for numerical optimization [13-19]. Recently, stochastic approaches have also been proposed $[20,21]$.

Although several model updating techniques have been developed and implemented in the field of structural mechanics, in the multibody field the issue of model updating has been just marginally addressed. Indeed, most of the works proposed in the literature on model updating address structures and often the proposed methods are suitable for structures with a few degrees of freedom. The resulting methods are, therefore, not effective for mechanisms which have some peculiar features. On the contrary, researches in the field of multibody systems are more frequently targeted to the development of efficient model formulations, by assuming that exact model parameters are known.

To solve this open issue, this paper introduces a model updating approach for flexible-link mechanisms based on the equivalent rigid-link system (ERLS) $[8,9]$ and formulated through ordinary differential equations (ODEs), which is a common method adopted for dynamic modeling. The main goal of this paper is to formulate a comprehensive approach that exploits experimental mode shapes and eigenfrequencies obtained through modal analysis, in order to identify the correct values of the model parameters. The formulation of an effective approach imposes several steps that are carefully accounted for in this work. Some preliminary results have been proposed by the authors in conference paper [22], which outlines the method idea through a simplified approach. The method is here extended to deal with a larger number of vibrational modes and constraints through an improved formulation which ensures more precise results and accounts for more measurements: not only the proposed approach is capable of exploiting more modal data, but also it takes advantage of information on the static behaviour of the mechanism. Additionally, some effective numerical methods are here proposed to speed up the problem formulation and solutions even in the presence of large dimensional models and a larger number of measured vibrational modes.

The remainder of the paper is organized as follows. In Section 2, the modeling of FLMs based on the ERLS is briefly recalled, as well as model linearization. In Section 3, some arrangements have been discussed to make experimental data compatible with model coordinates. In Section 4, the proposed numerical technique is introduced and thoroughly discussed. In Section 5, the experimental validation of the method is proposed by applying the method to a lightweight planar manipulator. Concluding remarks are given in Section 6.

\section{Model Based on the Equivalent Rigid-Link System}

2.1. Definitions. The paper deals with FLMs with scleronomous and holonomic constraints. The motion of each link can be notionally written as the large rigid-body motion of an equivalent rigid-link system (ERLS) plus the small elastic displacements with respect to the ERLS itself $[8,9]$. Basically, the ERLS represents a moving reference from which the elastic displacements are defined. Such an approach allows for a simple formulation and solution of the nonlinear differential equations governing the system motion. Indeed, once the mutual dynamic coupling between the ERLS and the elastic displacements is correctly accounted for in the model, the ERLS can be represented by taking advantage of the kinematics of rigid multibody systems as a function of its generalized coordinates q. Additionally, if the ERLS is placed very close to the actual deformed mechanism, then the small displacement assumption is correct and the classical FEs can be adopted.

The absolute position vector $\mathbf{b}_{i}$ of the nodes belonging to the $i$ th FE can be expressed with reference to a global reference frame as the sum of two contributions:

$$
\mathbf{b}_{i}=\mathbf{r}_{i}+\mathbf{u}_{i},
$$

where $\mathbf{u}_{i}$ is the vector of the nodal elastic displacements of the $i$ th element expressed with respect to the ERLS, and $\mathbf{r}_{i}$ is the vector of the nodal positions of the $i$ th element of the ERLS and it is a function of the ERLS generalized coordinates $\mathbf{q}$, that is, it can be computed through the rigidbody kinematics. A schematic representation of such definitions is provided in Figure 1.

Let $\mathbf{u}$ be the vector collecting the elastic displacement of all the nodes and $\mathbf{b}$ be the vector of the positions of all the nodes of the FLM, then (1) leads to the following expression that holds in the case of infinitesimal displacements $\mathrm{d} \mathbf{b}, \mathrm{d} \mathbf{r}$, and du:

$$
\mathrm{d} \mathbf{b}=\mathrm{d} \mathbf{r}+\mathrm{d} \mathbf{u}=\left[\begin{array}{ll}
\mathbf{I} & \mathbf{S}
\end{array}\right]\left\{\begin{array}{l}
\mathrm{d} \mathbf{u} \\
\mathrm{d} \mathbf{q}
\end{array}\right\},
$$

where $\mathbf{I}$ is the identity matrix and $\mathbf{S}$ is the sensitivity coefficient matrix for all the nodes of the ERLS. Matrix [ I S ] is not square since it has $n_{u^{\prime}}$ rows and $n_{u^{\prime}}+n_{q}$ columns, with $n_{u^{\prime}}$ and $n_{q}$ being the numbers of the elastic dofs and of the ERLS generalized coordinates, respectively. This means that the linear system in (2) is underdetermined. To overcome

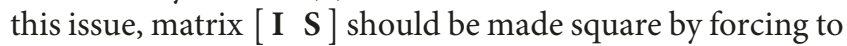
zero $n_{q}$ elements of $\mathbf{u}$. In this way, the system in (2) is 


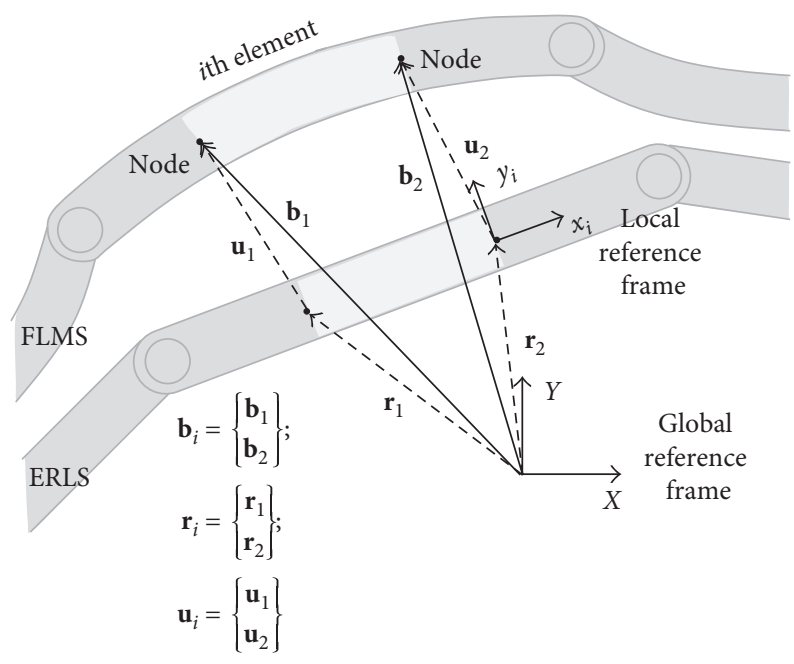

FIgURE 1: ERLS definitions.

determined and hence the position of the ERLS is defined univocally with respect to the actual deformed mechanism.

2.2. The Nonlinear Model. The final expression of the system equations of motion is given by the following set of nonlinear ordinary differential equations (ODEs):

$$
\begin{aligned}
& {\left[\begin{array}{cc}
\mathbf{M}_{\mathrm{e}} & \mathbf{M}_{\mathrm{e}} \mathbf{S} \\
\mathbf{S}^{T} \mathbf{M}_{\mathrm{e}} & \mathbf{S}^{T} \mathbf{M}_{\mathrm{e}} \mathbf{S}
\end{array}\right]\left\{\begin{array}{l}
\ddot{\mathbf{u}}(t) \\
\ddot{\mathbf{q}}(t)
\end{array}\right\}+\left[\begin{array}{cc}
2 \mathbf{M}_{\mathrm{G}}+\mathbf{C}_{\mathrm{e}} & -\mathbf{M}_{\mathrm{e}} \dot{\mathbf{S}} \\
\mathbf{S}^{T} 2 \mathbf{M}_{\mathrm{G}} & \mathbf{S}^{T} \mathbf{M}_{\mathrm{e}} \dot{\mathbf{S}}
\end{array}\right]\left\{\begin{array}{c}
\dot{\mathbf{u}}(t) \\
\dot{\mathbf{q}}(t)
\end{array}\right\}} \\
& +\left[\begin{array}{cc}
\mathbf{K}_{\mathrm{e}} & 0 \\
\mathbf{0} & \mathbf{0}
\end{array}\right]\left\{\begin{array}{c}
\mathbf{u}(t) \\
\mathbf{q}(t)
\end{array}\right\}=\left[\begin{array}{c}
\mathbf{I} \\
\mathbf{S}^{T}
\end{array}\right]\{\mathbf{v}(t)\},
\end{aligned}
$$

$\mathbf{M}_{\mathrm{e}}=\mathbf{M}_{\mathrm{e}}(\mathbf{q}), \mathbf{C}_{\mathrm{e}}=\mathbf{C}_{\mathrm{e}}(\mathbf{q})$, and $\mathbf{K}_{\mathrm{e}}=\mathbf{K}_{\mathrm{e}}(\mathbf{q})$ are obtained by assembling the mass, damping, and stiffness matrices of all the FEs adopted to model the link; $\mathbf{M}_{\mathrm{G}}=\mathbf{M}_{\mathrm{G}}(\mathbf{q}, \dot{\mathbf{q}})$ collects the link centrifugal and Coriolis effects. Finally, $\mathbf{v}$ is the vector of the nodal external forces and torques. The offdiagonal terms in the matrices in (3) represent the coupling between the ERLS generalized coordinates and the elastic displacements.

2.3. The Linearized Model. The use of linearized models is very satisfactory in the case of small deformations about operating points and hence can be successfully used in the synthesis of control schemes and state observers [23, 24]. Additionally, mode shapes and eigenfrequencies can be easily computed. As for the use of a minimal set of ODEs for the model synthesis, despite the large use of differential algebraic equations (DAEs) for modeling FLMs [25], it is justified by the possibility to obtain the exact spectrum. Indeed, models employing DAEs do not allow for the straightforward use of such a kind of analysis, and the spectrum of the linearized model is affected by the linearization method [26].

Linearization assumes small displacements about a static equilibrium configuration, which is set by the equilibrium configuration of the ERLS $\mathbf{q}_{\mathrm{e}}$. Then the following linear model is obtained:

$$
\begin{aligned}
& {\left[\begin{array}{cc}
\mathbf{M}_{\mathrm{e}} & \mathbf{M}_{\mathrm{e}} \mathbf{S} \\
\mathbf{S}^{T} \mathbf{M}_{\mathrm{e}} & \mathbf{S}^{T} \mathbf{M}_{\mathrm{e}} \mathbf{S}
\end{array}\right]_{\mathbf{q} \equiv \mathbf{q}_{\mathrm{e}}}\left\{\begin{array}{c}
\ddot{\mathbf{u}}(t) \\
\ddot{\mathbf{q}}(t)
\end{array}\right\}+\left[\begin{array}{cc}
\mathbf{C}_{\mathrm{e}} & \mathbf{0} \\
\mathbf{0} & \mathbf{0}
\end{array}\right]_{\mathbf{q} \equiv \mathbf{q}_{\mathrm{e}}}\left\{\begin{array}{c}
\dot{\mathbf{u}}(t) \\
\dot{\mathbf{q}}(t)
\end{array}\right\}} \\
& +\left[\begin{array}{cc}
\mathbf{K}_{\mathrm{e}} & \mathbf{0} \\
\mathbf{0} & -\left(\frac{\mathrm{d}\left(\mathbf{S}^{T} \mathbf{M}_{\mathrm{e}}\right)}{\mathrm{d} \mathbf{q}} \otimes \mathbf{g}+\frac{\mathrm{d} \mathbf{S}^{T}}{\mathrm{~d} \mathbf{q}} \otimes \mathbf{v}_{\mathrm{e}}\right)
\end{array}\right]_{\mathbf{q} \equiv \mathbf{q}_{\mathrm{e}}}\left\{\begin{array}{l}
\mathbf{u}(t) \\
\mathbf{q}(t)
\end{array}\right\} \\
& =\left[\begin{array}{c}
\mathbf{I} \\
\mathbf{S}^{T}
\end{array}\right]_{\mathbf{q} \equiv \mathbf{q}_{\mathrm{e}}}\{\mathbf{v}\} .
\end{aligned}
$$

In (4), $\mathbf{g}$ is the gravity acceleration vector. Matrices $\mathbf{M}_{\mathrm{e}}$, $\mathbf{S}, \mathbf{K}_{\mathrm{e}}$ and the derivatives $\mathrm{d}\left(\mathbf{S}^{T} \mathbf{M}_{\mathrm{e}}\right) / \mathrm{d} \mathbf{q}$ and $\mathrm{d} \mathbf{S}^{T} / \mathrm{d} \mathbf{q}$ are computed about the equilibrium configuration. The product $\left(\mathrm{d} \mathbf{S}^{T} / \mathrm{d} \mathbf{q}\right) \otimes \mathbf{v}_{\mathrm{e}}$ denotes the inner product of matrix $\left[\partial S_{i, 1} / \partial q_{j} \cdots \partial S_{i, n} / \partial q_{j}\right]$ with vector $\mathbf{v}_{\mathrm{e}}$, for all the subscripts $i, j$ (analogous meaning for $\left.\left(\mathrm{d}\left(\mathbf{S}^{T} \mathbf{M}_{\mathrm{e}}\right) / \mathrm{d} \mathbf{q}\right) \otimes \mathbf{g}\right)$.

Since model updating should be performed by investigating the system in an asymptotically stable equilibrium configuration, gravity should be compensated in the experimental modal analysis whenever the mechanism lies in the vertical plane. Indeed, the absence of a stable equilibrium would make the mechanism diverge from the initial configuration after the excitations, and therefore, the system dynamics would not meet the basic assumption beneath the linearized model. A possible approach is balancing through external springs that determine asymptotic stability of the equilibrium point. The stiffness of these springs should be included in the stiffness matrix and among the updating variables. In contrast, the use of brakes, for example, those employed in the motors, changes the boundary conditions. Therefore, model updating may lead to less reliable results.

\section{Data Consistency between Model and Experimental Modal Analysis}

3.1. Size Compatibility between the Measured Mode Shapes and the Model. Model updating procedures need one-to-one correspondence between model coordinates and measured mode shapes. In practical applications, this requirement is usually not satisfied because of the physical inaccessibility to some locations or for difficulties in the measurement of some dofs (such as rotational dofs). This is often denoted as spatial incompleteness. Therefore, the experimental mode shapes are usually not compatible with the FE model, and hence, as a preliminary step, the size of either model or the measured data should be modified to meet this requirement. Two strategies are usually adopted to obtain the one-to-one correspondence: reducing the FE model by removing the unmeasured dofs or expanding the experimental results by estimating the displacement of such unmeasured variables. However, model reduction might negatively affect the model accuracy. Hence, to avoid losses of information, the method 
proposed in this work expands the experimental data to match the dimension of the FE model by means of transformation matrices $\mathbf{T}_{i} \in \mathbb{R}^{n_{u} \times n_{u}^{*}}$ that maps the $n_{u}^{*}$-dimensional measured eigenvectors into a $n_{u}$-dimensional one that fits the number of model dofs $\left(n_{u}>n_{u}^{*}\right)$ :

$$
\overline{\boldsymbol{\eta}}_{i}=\mathbf{T}_{i} \overline{\boldsymbol{\eta}}_{i}^{*}
$$

where $\overline{\boldsymbol{\eta}}_{i}^{*}$ denotes the ith measured eigenvector while $\overline{\boldsymbol{\eta}}_{i}$ denotes the expanded one.

The use of curve fitting, by means of the shape functions of the FEs and a set of redundant measurements of accessible dofs, is a reliable way to handle experimental data contaminated by noise. Alternatively, spline functions can be adopted for high-frequency modes, even if they are affected by errors due to measurements or to the mode-extraction algorithm. Section 5.3 provides a more detailed explanation on the procedure in the case of the planar mechanism studied here.

\subsection{Mode Shape Transformation from the Physical Reference} Frame to One of the Models. After expansion, the measured mode shapes should be transformed from the physical reference frame adopted for experimental measurements to the fictitious one used in the ERLS-based FE model. Indeed, sensors employed to perform experimental tests typically provide measurements with respect to local reference frames corresponding to the mechanism in its initial static configuration. Conversely, the model elastic displacements (collected in $\mathbf{u}$ ) are defined with respect to the ERLS and are expressed in a global reference frame (Figure 1). Hence, some of the elastic displacements are forced to zero to make the ERLS follow the actual elastic mechanism. At the same time, the ERLS coordinates should be included in the displacement vector and therefore in the eigenvectors.

Let $\mathbf{q}_{\mathrm{e}}$ be the static equilibrium configuration corresponding to the actual position of the mechanism, adopted for both the experimental modal analysis and the model linearization. Let us assume that the mechanism is vibrating in accordance with the $i$ th mode about the equilibrium configuration. The absolute position of the nodes of the FEM, denoted $\mathbf{b}_{i}$, can be represented both through the eigenvector defined in the physical frame adopted for the experimental analysis, $\bar{\eta}_{i}$, and through the one defined with the model coordinates, $\boldsymbol{\eta}_{i}$. If $\mathbf{b}_{i}$ is defined in a global frame, the eigenvector measured in the physical frame should be rotated onto the global reference frame through the ERLS kinematic constraint equations:

$$
\mathbf{b}_{i}=\overline{\mathbf{r}}\left(\mathbf{q}_{\mathrm{e}}\right)+\boldsymbol{\Theta}\left(\mathbf{q}_{\mathrm{e}}\right) \overline{\boldsymbol{\eta}}_{i},
$$

where $\bar{r}$ is the vector of the nodal position of the FLM in the configuration set by $\mathbf{q}_{\mathrm{e}}$ while $\Theta$ is a block transformation matrix which depends on the rigid-body kinematic constraints.

If, in contrast, the same configuration is represented through the eigenvector of the linearized model $\boldsymbol{\eta}_{i}$ expressed with respect to the ERLS at configuration $\mathbf{q}_{i}=\mathbf{q}_{\mathrm{e}}+\Delta \mathbf{q}$, the following relation holds:

$$
\mathbf{b}_{i}=\mathbf{r}\left(\mathbf{q}_{i}\right)+\mathbf{\eta}_{i}
$$

where $\mathbf{r}\left(\mathbf{q}_{i}\right)$ is the vector of the nodal position of the ERLS in the configuration set by $\mathbf{q}_{i}$. The displacement of the ERLS-generalized coordinates $\Delta \mathbf{q}$ represents the rotation of the ERLS, which moves to follow the actual mechanism. Hence, it is equal to the $n_{q}$ zeroed elements of $\mathbf{u}$ discussed in Section 2.1.

A graphical representation of (6) and (7) is provided in Figure 2 with reference to a single flexible link.

The final transformation to make the experimental eigenvectors consistent with the model formulation can be inferred from (6) and (7)

$$
\widehat{\boldsymbol{\eta}}_{i}=\overline{\mathbf{r}}\left(\mathbf{q}_{\mathrm{e}}\right)-\mathbf{r}\left(\mathbf{q}_{i}\right)+\boldsymbol{\Theta}\left(\mathbf{q}_{\mathrm{e}}\right) \overline{\boldsymbol{\eta}}_{i},
$$

where $\widehat{\eta}_{i}$ denotes the transformed experimental eigenvector to be employed in the model updating procedure.

The result proposed in (8) is the exact relation that transforms the measured expanded eigenvectors and is an improvement of the approximated one proposed in [22].

\section{The Model Updating Technique}

4.1. Formulation of the Mass and Stiffness Matrix Updating. The model is updated by firstly correcting the inertial and elastic parameters through the undamped model. This choice simplifies the formulation of the inverse eigenvalue problem by casting it as a first-order problem. Hence, real eigenpairs are obtained, thus improving the numerical solution by just handling real numbers. The identification of damping matrix can be performed separately, through the well-established methods proposed in literature [27, 28], once the inertial and elastic parameters have been updated. A "two-step" approach, that updates stiffness and the mass matrices first and then updates damping matrix, is often adopted in literature since it is usually more reliable [29]. The negligible internal damping of beams further corroborates this approach.

The linearized undamped model in (4) is represented through the mass $\mathbf{M}^{O} \in \mathbb{R}^{N \times N}$ and the stiffness $\mathbf{K}^{O} \in \mathbb{R}^{N \times N}$ matrices, which are the initial (nominal) system matrices based on the nominal inertial and elastic parameters ( $N$ is number of model dofs, including both rigid and elastic coordinates: $N=n_{q}+n_{u}$ ). The updating of the model matrices is represented here through additive correction matrices, the so-called update matrices $\Delta \mathbf{M} \in \mathbb{R}^{N \times N}$ and $\Delta \mathbf{K} \in \mathbb{R}^{N \times N}$, which collect the parameters that are affected by uncertainty and hence should be corrected. Therefore, the mass and stiffness matrices of the $N$-dimensional updated model are $\mathbf{M}^{O}+\Delta \mathbf{M}$ and $\mathbf{K}^{O}+\Delta \mathbf{K}$. The topology of the update matrices depends on the topology of $\mathbf{M}^{O}$ and $\mathbf{K}^{O}$.

Following a popular representation, $\Delta \mathbf{M}$ and $\Delta \mathbf{K}$ can be written as the sum of the update matrices related to each update (unknown) parameter, denoted $\Delta \mathbf{M}_{h}$ and $\Delta \mathbf{K}_{h}$, that represent the effect of each additive update parameter: 


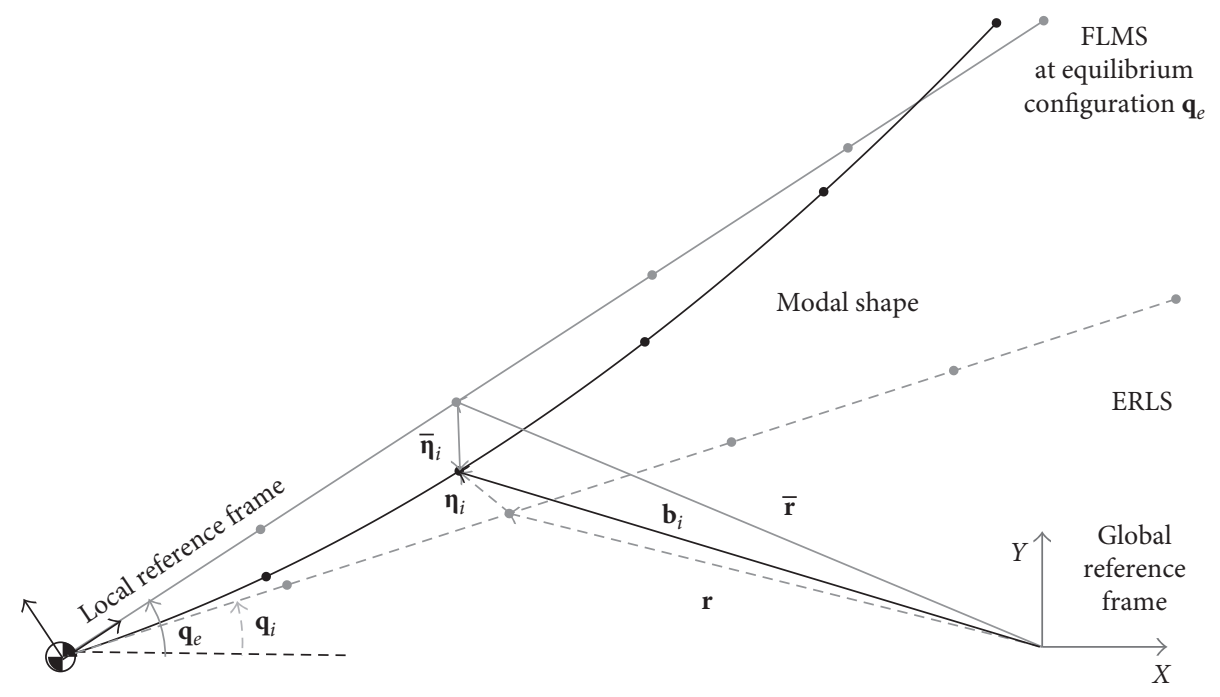

FIGURE 2: Interpretation of the analytical and experimental eigenvectors.

$$
\begin{aligned}
& \Delta \mathbf{M}=\sum_{h=1}^{N_{m}} \Delta \mathbf{M}_{h}, \\
& \Delta \mathbf{K}=\sum_{h=1}^{N_{k}} \Delta \mathbf{K}_{h} .
\end{aligned}
$$

The method proposed in this paper updates masses and stiffnesses simultaneously to address their coupling effects more effectively and hence obtaining more reliable results. In contrast, several works in literature proposes two-step approaches that split mass and stiffness updating in two sequential steps. The simultaneous updating imposes to tackle the different magnitudes of masses, inertias, and elastic parameters that cause ill-conditioning of the numerical problem if they are not properly accounted for. Hence, to improve the problem conditioning, dimensionless (normalized) modifications $\bar{m}_{h}$ and $\bar{k}_{h}$ are introduced:

$$
\begin{gathered}
\bar{m}_{h}=\frac{\Delta m_{h}}{m_{h}^{O}}, \\
\bar{k}_{h}=\frac{\Delta k_{h}}{k_{h}^{O}},
\end{gathered}
$$

$\Delta m_{h}$ and $\Delta k_{h}$ are the corrections on the parameters of the original model ( $m_{h}^{O}$ and $k_{h}^{O}$, resp.) that should be updated:

$$
\begin{gathered}
m_{h}=m_{h}^{O}+\Delta m_{h}, \\
k_{h}=k_{h}^{O}+\Delta k_{h} .
\end{gathered}
$$

For the parameters whose original value is zero, the reference value might be defined as the upper bound of their allowed modifications. Nonetheless, other arbitrary reference numbers can be assumed, though reasonable. All the variables $\bar{m}_{j}$ and $\bar{k}_{h}$ are collected in the $\left(N_{m}+N_{k}\right)$-dimensional vector of the updating parameters $\chi=\left\{\bar{m}_{1}, \ldots, \bar{m}_{N_{m}} \bar{k}_{1}, \ldots, \bar{k}_{N_{k}}\right\}^{T}$, which collects all the problem unknowns $\left(N_{m}\right.$ and $N_{k}$ denote the number of parameters that can be updated in the mass and stiffness matrix, resp.).

Equation (9) can be written in the following form by introducing the Jacobian matrices $\mathbf{J}_{m_{h}}$ and $\mathbf{J}_{k_{h}}$ :

$$
\begin{aligned}
\Delta \mathbf{M} \simeq \sum_{h=1}^{N_{m}} m_{h}^{O} \mathbf{J}_{m_{h}} \bar{m}_{j}, \\
\Delta \mathbf{K} \simeq \sum_{h=1}^{N_{k}} k_{h}^{O} \mathbf{J}_{k_{h}} \bar{k}_{h},
\end{aligned}
$$

where

$$
\begin{aligned}
& \mathbf{J}_{m_{h}}=\left[\frac{\partial \mathbf{M}^{O}}{\partial m_{h}^{O}}\right], \\
& \mathbf{J}_{k_{h}}=\left[\frac{\partial \mathbf{K}^{O}}{\partial k_{h}^{O}}\right] .
\end{aligned}
$$

The representation in (12) is exact, and Jacobians are invariant whenever the updating parameters linearly affect the mass and stiffness matrices, such as in the case of nodal masses, inertias, or springs, and also in the case of Young's modulus, mass density, and sectional area of the beams. If, in contrast, the Jacobians are not constant (such as in the case of including the link lengths as the unknown parameters), iterations should be performed.

The formulation through the Jacobian matrices allows for the use of some numerical methods to formulate the model updating problem, by speeding up the computation and reducing the need of complicate analytical developments. More details are provided in Section 5.

4.2. Formulation of the Inverse Eigenproblem. The formulation of the model updating method is based on the following eigenvalue problem that must hold for any measured eigenpairs $\left(\widehat{\omega}_{i}^{2}, \widehat{\boldsymbol{\eta}}_{i}\right), i=1, \ldots, n$ (where $n \leq N$ is the number 
of measured modes and $\widehat{\omega}_{i}$ is the natural frequency associated to eigenvector $\widehat{\boldsymbol{\eta}}_{i}$ ):

$$
\widehat{\omega}_{i}^{2} \widehat{\boldsymbol{\eta}}_{i}^{\mathrm{T}}\left[\mathbf{M}^{O}+\mathbf{\Delta} \mathbf{M}\right] \widehat{\boldsymbol{\eta}}_{i}-\widehat{\boldsymbol{\eta}}_{i}^{\mathrm{T}}\left[\mathbf{K}^{O}+\mathbf{\Delta} \mathbf{K}\right] \widehat{\boldsymbol{\eta}}_{i}=0 .
$$

Any arbitrary normalization of the measured eigenvectors can be adopted.

The formulation of the eigenvalue problem in the form of (14), rather than the form $\widehat{\omega}_{i}^{2}\left[\mathbf{M}^{O}+\Delta \mathbf{M}\right] \widehat{\boldsymbol{\eta}}_{i}-\left[\mathbf{K}^{O}+\Delta \mathbf{K}\right] \widehat{\eta}_{i}$ $=\mathbf{0}$, has the advantage that just one equation is written for each vibrational modes, thus improving numerical conditioning.

By introducing the representation of the update matrices given in (12), the scalar equations in (14) are written as linear problems in the unknown $\left\{\bar{m}_{j}, \bar{k}_{h}\right\}$ :

$$
\begin{gathered}
\widehat{\boldsymbol{\eta}}_{i}^{T}\left[\widehat{\omega}_{i}^{2} \mathbf{M}^{O}-\mathbf{K}^{O}\right] \widehat{\boldsymbol{\eta}}_{i}+\widehat{\omega}_{i}^{2} \boldsymbol{\eta}_{i}^{T} \sum_{h=1}^{N_{m}} m_{h}^{O} \mathbf{J}_{m_{h}} \widehat{\boldsymbol{\eta}}_{i} \bar{m}_{h} \\
-\boldsymbol{\eta}_{i}^{T} \sum_{h=1}^{N_{k}} k_{h}^{O} \mathbf{J}_{k_{h}} \widehat{\boldsymbol{\eta}}_{i} \bar{k}_{h}=0 .
\end{gathered}
$$

Equation (15) can be represented in the typical compact form of a linear algebraic system through vector $\mathbf{a}_{i}$, which collects the known coefficients, and scalar $d_{i}=-\widehat{\boldsymbol{\eta}}_{i}^{T}\left[\widehat{\omega}_{i}^{2} \mathbf{M}^{O}-\mathbf{K}^{O}\right] \widehat{\boldsymbol{\eta}}_{i}$, which is the known-term that depends on the original model to be updated and on the experimental data:

$$
\mathbf{a}_{i}^{T} \mathbf{\chi}-d_{i}=0 .
$$

Since the problem unknowns should be constrained by a feasible set and the condition in (16) should be simultaneously satisfied for all the measured eigenpairs $(i=1, \ldots, n)$, an exact solution might not exist. In practice, the measured eigendata are usually not reproduced exactly by the updated model.

Therefore, it is more reasonable approximating the updating problem as a least-square minimization:

$$
\min _{\mathbf{x}}\|\mathbf{W}(\mathbf{A} \mathbf{x}-\mathbf{d})\|_{2}^{2}
$$

$\mathbf{A} \in \mathbb{R}^{n \times\left(N_{m}+N_{k}\right)}$ and $\mathbf{d} \in \mathbb{R}^{n}$ collect the terms of each eigenvalue problem in (16): the rows of matrix $\mathbf{A}$ are the vectors $\mathbf{a}_{i}$, while the entries of $\mathbf{d}$ are the scalars $d_{i}$. The diagonal matrix $\mathbf{W} \in \mathbb{R}^{n \times n}$ is defined to weigh the residual of each eigenvalue problem through the positive and scalar weights $w_{i}$, with $i=1, \ldots, n$. $\mathbf{W}$ allows giving less importance to those eigenpairs whose measurements are less reliable, as well as to give higher importance to the vibrational modes that are more observable and controllable.

Regularization can also be adopted to penalize some of the components of $\chi$ and to improve the numerical conditioning. Indeed, some model parameters might be more uncertain than the others, and therefore, it is more desirable that the updating procedure modifies them. Therefore, the Tikhonov's regularization term $\lambda\|\Omega \chi\|_{2}^{2}$ is included in (19). The positive scalar $\lambda$ is named the regularization parameter and trades-off between the cost of missing the eigenpair specifications (16) and the cost of using large values of the unknown variables (i.e., $\|\chi\|_{2}^{2}$ ). An effective way to select $\lambda$ is the use of the $L$-curve method for the unconstrained problem, which admits analytical solution and hence the calculation is straightforward. According to [30], $\lambda$ is chosen as the value that corresponds to the point at the "vertex" of the curve (whose shape recalls the letter "L"), that is, the point with the largest magnitude curvature.

The positive-definite real matrix $\boldsymbol{\Omega} \in \mathbb{R}^{\left(N_{m}+N_{k}\right) \times\left(N_{m}+N_{k}\right)}$ is the regularization operator and can be adopted to define the relative weight between the updates of the different model parameters. The model updating problem therefore becomes

$$
\min _{\mathbf{\chi}}\left(\|\mathbf{W}(\mathbf{A} \mathbf{X}-\mathbf{d})\|_{2}^{2}+\lambda\|\mathbf{\Omega} \chi\|_{2}^{2}\right)
$$

The numerical solution of the minimization problem should be further improved through preconditioning to handle effectively large dimensional matrices and the simultaneous presence of low-frequency and high-frequency vibrational modes. By using the following preconditioner, $\left(\mathbf{A}^{T} \mathbf{W}^{T} \mathbf{W} \mathbf{A}+\lambda \boldsymbol{\Omega}\right)^{-1} \mathbf{A}^{T} \mathbf{W}^{T}$, the system in (17) is written in the following equivalent form whose condition number is one:

$$
\min _{\boldsymbol{X}}\left\|\boldsymbol{X}-\left(\mathbf{A}^{T} \mathbf{W}^{T} \mathbf{W} \mathbf{A}+\lambda \boldsymbol{\Omega}\right)^{-1} \mathbf{A}^{T} \mathbf{W}^{T} \mathbf{W} \mathbf{d}\right\|_{2}^{2} .
$$

A wise selection of $\lambda$ also ensures that the inverse $\left(\mathbf{A}^{T} \mathbf{W}^{T} \mathbf{W} \mathbf{A}+\lambda \boldsymbol{\Omega}\right)^{-1}$ is well conditioned, and hence the optimization problem in (19) is correctly formulated. Therefore, more reliable solutions can be achieved compared to [22] that effectively handle a larger number of vibrational modes over a larger frequency range.

4.3. Static Equilibrium Constraints. The knowledge of the static equilibrium configuration allows for the inclusion of some additional information that can be handled by means of the optimization-based formulation proposed in this work. In such a rest configuration, the mechanism is subjected to the action of gravity forces (whenever it lies on the vertical plane), friction, elastic forces due to balancing springs (connecting the mechanism to the ground or linking more links), and forces exerted by actuators. In the case of small elastic displacement with respect to the ERLS, which is the basic assumption that has been adopted in the development of such a model and therefore is taken all along the paper, the effect of the elastic deformation of the links can be neglected in the computation of the aforementioned forces [8]. Hence, a rigid-body model can be assumed, and the mechanism coincides with the ERLS.

The application of the principle of virtual work for the rigid-link system leads to the following equation:

$$
\delta \mathbf{r}^{T}\left(\mathbf{M}_{\mathrm{e}}+\Delta \mathbf{M}_{\mathrm{e}}\right) \mathbf{g}+\delta \mathbf{r}^{T}\left(\mathbf{v}_{\text {spring }}+\mathbf{v}_{\text {control }}+\mathbf{v}_{\text {friction }}\right)=0,
$$

where $\delta \mathbf{r}^{T}$ is the vector of the virtual displacements of all the nodes of the ERLS. Vectors $\mathbf{v}_{\text {spring }}, \mathbf{v}_{\text {control }}$, and $\mathbf{v}_{\text {friction }}$ collect, respectively, the spring, control, and friction nodal forces (i.e., $\mathbf{v}_{\text {spring }}+\mathbf{v}_{\text {control }}+\mathbf{v}_{\text {friction }}=\mathbf{v}$ ). $\Delta \mathrm{M}_{\mathrm{e}}$ is the 
$n_{u}$-dimensional submatrix of matrix $\Delta \mathbf{M}$ that collects the modification of $\mathbf{M}_{\mathrm{e}}$. For clarity of explanation, the effect of the external springs has been represented in (20) through $\mathbf{v}_{\text {spring }}$ (rather than using stiffness matrix), which also collects some updating unknowns.

Equation (20) can be transformed into a set of $n_{q}$ independent equations (i.e., one equation for each ERLS dof), through the matrix of the sensitivity coefficients:

$$
\mathbf{S}^{T}\left(\mathrm{M}_{\mathrm{e}}+\Delta \mathrm{M}_{\mathrm{e}}(\chi)\right) \mathbf{g}+\mathbf{S}^{T}\left(\mathbf{v}_{\text {spring }}(\chi)+\mathbf{v}_{\text {control }}+\mathbf{v}_{\text {friction }}\right)=\mathbf{0} .
$$

All the matrices are computed at the static equilibrium configuration adopted for linearization, $\mathbf{q}=\mathbf{q}_{\mathrm{e}}$.

Equation (21) should be included in model updating as equality constraints, which are linear functions of the problem unknown $\chi$ :

$$
\mathbf{H} \chi \mathbf{~}=\mathbf{h},
$$

$\mathbf{H}$ and $\mathbf{h}$ collect the coefficients of the linear system.

The inclusion of (22) into the minimization problem has some practical issues since the exact value of friction is usually unknown. If reasonable upper bounds of friction are estimated, the friction forces reflected to the ERLS coordinates $\mathbf{S}^{T} \mathbf{v}_{\text {friction }}$, can be treated as uncertain values that should be bounded, $\left|\mathbf{S}^{T} \mathbf{v}_{\text {friction }}\right| \leq \boldsymbol{v}$. Therefore, the $n_{q}$ equality constraints in (22) can be relaxed as $2 n_{q}$ inequality constraints:

$$
\begin{aligned}
& \mathbf{H} \boldsymbol{\chi} \leq \mathbf{h}_{\mathrm{U}} \\
& \mathbf{H} \boldsymbol{\chi} \geq \mathbf{h}_{\mathrm{L}} .
\end{aligned}
$$

4.4. Constraints on the Updating Parameters. The correction of parameters should be bounded to ensure physical meaning. Hence, lower and upper bounds on each parameter (denoted as $\chi_{\mathrm{L}}$ and $\chi_{U}$, resp.) and on combinations of more parameters should be defined. The latter type of constraint is defined through a convex polyhedron (by means of a matrix $\mathbf{G}$ and a vector $\gamma$ ). These two types of constraint define the following convex feasible set $\Gamma$ (the inequalities are element-wise):

$$
\Gamma=\left\{\chi \mid\left(\chi_{L} \leq \chi \leq \chi_{U}\right) \cap G \chi \leq \gamma\right\} .
$$

4.5. Formulation of the Model Updating Problem. The model updating problem is finally represented as the following constrained minimization problem:

$$
\begin{array}{ll}
\min _{\boldsymbol{\chi}} & \left\|\boldsymbol{\chi}-\left(\mathbf{A}^{T} \mathbf{W}^{T} \mathbf{W} \mathbf{A}+\lambda \boldsymbol{\Omega}\right)^{-1} \mathbf{A}^{T} \mathbf{W}^{T} \mathbf{W} \mathbf{d}\right\|_{2}^{2}, \\
\text { such that } & \left\{\begin{array}{l}
\mathbf{H} \boldsymbol{\chi} \leq \mathbf{h}_{U}, \\
\mathbf{H} \boldsymbol{\chi} \geq \mathbf{h}_{\mathrm{L}}, \\
\boldsymbol{\chi} \in \Gamma .
\end{array}\right.
\end{array}
$$

Both the objective function and the feasible domain are convex. Hence, the solution is straightforward since convexity ensures that the global optimal solution can be found regardless of the selection of the initial guess.

In the case of medium-scale or large-scale problem, a convenient formulation is the one based on the quadratic programming optimization, rather than the least-square norm minimization in (25), that can be solved through reflective-trust-region algorithms:

$$
\begin{array}{ll}
\min _{\boldsymbol{\chi}} & \left\{\boldsymbol{\chi}^{T} \boldsymbol{\chi}-2\left(\left(\mathbf{A}^{T} \mathbf{W}^{T} \mathbf{W} \mathbf{A}+\lambda \boldsymbol{\Omega}\right)^{-1} \mathbf{A}^{T} \mathbf{W}^{T} \mathbf{W} \mathbf{d}\right)^{T} \boldsymbol{\chi}\right\}, \\
\text { such that } & \left\{\begin{array}{l}
\mathbf{H} \boldsymbol{\chi} \leq \mathbf{h}_{\mathrm{U}}, \\
\mathbf{H} \boldsymbol{\chi} \geq \mathbf{h}_{\mathrm{L}}, \\
\boldsymbol{\chi} \in \Gamma .
\end{array}\right.
\end{array}
$$

\section{Experimental Application}

5.1. Description of the Mechanism. The model updating strategy proposed in this paper has been applied to the manipulator with flexible links shown in Figure 3(a). Such a manipulator consists of five rods with circular cross sections made of aluminum anticorodal. The links are connected to each other by means of revolute joints, created by means of double ball bearings with negligible clearance and friction losses, inserted in aluminum housings. A serialparallel kinematic topology is obtained. Links 1,2, and 5 are driven by three brushless motors. The mechanism is equipped with two identical balancing springs, modeled as a single lumped spring, which has been introduced to compensate for gravity force.

The links have been modeled with two-node and sixdegree-of-freedom Euler-Bernoulli's beam elements to represent the planar motion of the mechanism. Overall, nine beam elements have been adopted (Figure 3(b)). Lumped masses and nodal inertias have been adopted to represent the joints and the motors. The resulting model has 27 elastic dofs, and three ERLS-generalized coordinates $\mathbf{q}=\left\{\begin{array}{lll}q_{1} & q_{2} & q_{3}\end{array}\right\}^{T}$ are the joint absolute rotations of the motors. The rigid moving reference is then defined by forcing the ERLS to coincide with the actual flexible mechanism at the rotation of these three joints. Hence, three elastic displacements with respect to the ERLS are forced to zero in the FLM model, in order to place the moving reference defined by the equivalent rigid mechanism correctly (Section 2).

Model updating has been done by investigating the mechanism in the stable equilibrium configuration with no motor torques (i.e., $\mathbf{v}_{\text {control }}=\mathbf{0}$ ), where gravity forces are exactly compensated by the balancing force exerted by the two external springs and by friction, which corresponds to $\mathbf{q}_{\mathbf{e}}=\left\{\begin{array}{lll}1.082 & 2.635 & 4.712\end{array}\right\}^{T}[\mathrm{rad}]$.

5.2. The Experimental Modal Analysis. Experimental modal analysis has been performed through impact tests. A series of frequency response functions (FRFs) have been measured through accelerometers, while an instrumented impact hammer has been adopted to exert the excitation forces. 


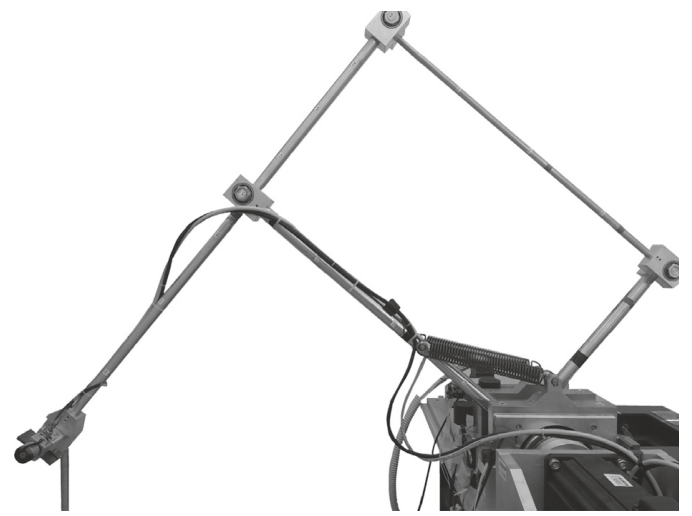

(a)

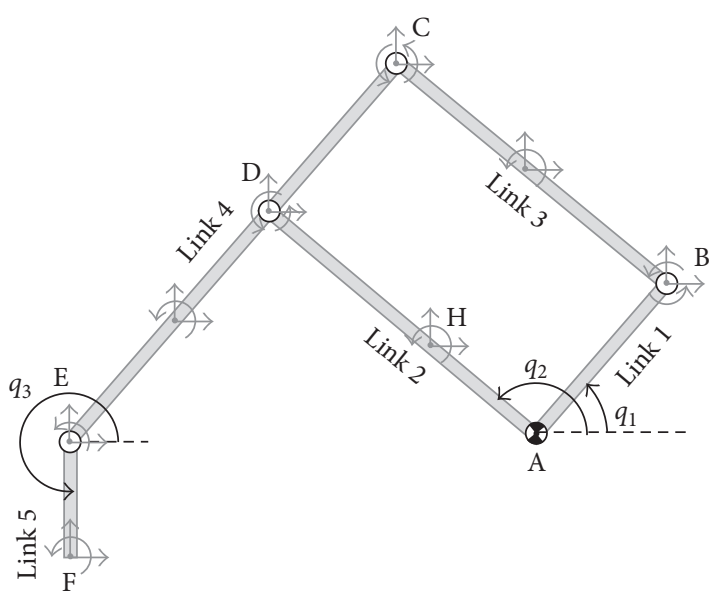

(b)

Figure 3: (a) Picture of the planar, three-dof manipulator and (b) its FE model.

The measurement chain consists in

(i) 5 accelerometers described in Table 1;

(ii) an instrumented impact hammer PCB Modally Tuned $086 C 03$ with teflon tip;

(iii) a front-end system LMS SCADAS Recorder (max input $\pm 10 \mathrm{~V}, \mathrm{ADC} 24 \mathrm{bit})$;

(iv) a modal software LMS Test.Lab Impact Testing 11B, with the following tools: Geometry, Modal Analysis, PolyMAX Modal Analysis.

The impact setup defined for the test is described in Table 2.

The number of measurement points has been selected to provide a reliable representation of the mode shapes in the frequency range of interest and for the interpolation to expand the experimental results to fit the model dimension with adequate smoothing of measurement noise and uncertainty (Section 5.3). In accordance with these aims, a manipulator geometry has been created through 22 points in the software tool LMS Test.Lab Impact Testing $11 B \mathrm{Ge}$ ometry, as shown in Figure 4.

As far as the frequency range of interest is concerned, it ranges from 0 to $200 \mathrm{~Hz}$. This interval corresponds, approximately, to the bandwidth of the motor current loop and hence to the range of frequencies where active control could be reasonably achieved. In contrast, the vibrational modes with higher frequency can neither be controlled nor excited in the forced motion, and hence they are not of interest for the model. Therefore, seven vibrational modes have been adopted to perform model updating. As a representative example of such FRFs, Figure 5 shows the 7 th row of the matrix of the FRFs, as it was provided by the software.

Measured data have been postprocessed using the PolyMAX of LMS Test.Lab $11 B$ tool, which extracts the mode shapes through the "pole-residual model" [31]. While eigenvalues can be easily identified with negligible uncertainty [32], the extraction of reliable mode shapes imposes careful and multiple execution of the test to get rid of measurement noise or wrong hammer excitations, as well as a wise use of the stabilization diagrams. A total number of 315 FRFs have been recorded by averaging four measurements. In such a way, a more reliable extraction of the modal parameters has been done and the effect of uncertainties introduced by the extraction algorithm can be neglected.

5.3. Size Compatibility between the FE Model and the Measurements. Least-square curve fitting has been employed to expand the set of measurements by estimating the unmeasured rotational dofs, denoted as $\varphi_{i}$ and collected in vector $\varphi$. Fitting is based on a redundant set of measured transverse displacements along the beams (Figure 4) and on the least-square regression of the measured displacements in the local frame along the links. The cubic polynomial interpolation shape functions of the Euler-Bernoulli's beam have been adopted, in accordance with the model described in Sections 2.2 and 5.1, which uses such a finite element. An undetermined linear system of the type in (27) is written for each link:

$$
A_{\varphi} \varphi=d_{\varphi}
$$

The size of the unknown vector $\varphi$ depends on the number of finite elements adopted in the model for such a link, while the number of equations (i.e., the number of lines of matrix of coefficients $\mathbf{A}_{\varphi}$ and of the known vector $\mathbf{d}_{\varphi}$ ) depends on the number of transverse displacements measured along the link.

5.4. Statement of the Model Updating Problem. The vector of the updating parameters, $\chi$, collects the following uncertain variables: the equivalent lumped stiffness of the balancing springs $\left(k_{\mathrm{bs}}\right)$, the nodal inertias $(J)$, masses $(M)$, the mass density of the links $(\rho)$, and the Young's modulus $(E)$. A different value of $E$ has been assumed for each link to account for different phenomena that result in local increase or decrease of the overall stiffness of the link. For example, local stiffening due to the kinematic joints and their coupling with the beams, as well as uncertainty on the actual beam 
TABLE 1: Accelerometers employed.

\begin{tabular}{lccc}
\hline Manufacturer & Model & Typology & $\begin{array}{c}\text { Sensitivity } \\
(\mathrm{mV} / \mathrm{g})\end{array}$ \\
\hline Endevco & $\begin{array}{c}\text { 27AM1-100 } \\
10203\end{array}$ & ICP & 102.4 \\
\hline Dytran & 3136A1945 & ICP & 100 \\
\hline PCB & 3741D4HB30G & Full-bridge DC & 66.7 \\
\hline Brüel \& Kjær & 4508 & ICP & 9.78 \\
Brüel \& Kjær & 4506 B triaxial & $\left\{\begin{array}{l}X \text {-axis ICP } \\
Y \text {-axis ICP }\end{array}\right.$ & 93.5 \\
& & $Z$-axis ICP & 98 \\
\hline
\end{tabular}

TABLE 2: Impact test settings.

\begin{tabular}{lc}
\hline Acquisition setting & \\
Sample frequency & $2048 \mathrm{~Hz}$ \\
Frequency resolution & $0.06 \mathrm{~Hz}$ \\
Acquisition time & $16 \mathrm{~s}$ \\
\hline Triggering & \\
Trigger level & $5.72 \mathrm{~N}$ \\
Pretrigger & $0.01 \mathrm{~s}$ \\
\hline Windowing & \\
Input & Uniform \\
Response & 4 \\
\hline Measurement averages & Exponential (decay 90\%) \\
\hline
\end{tabular}

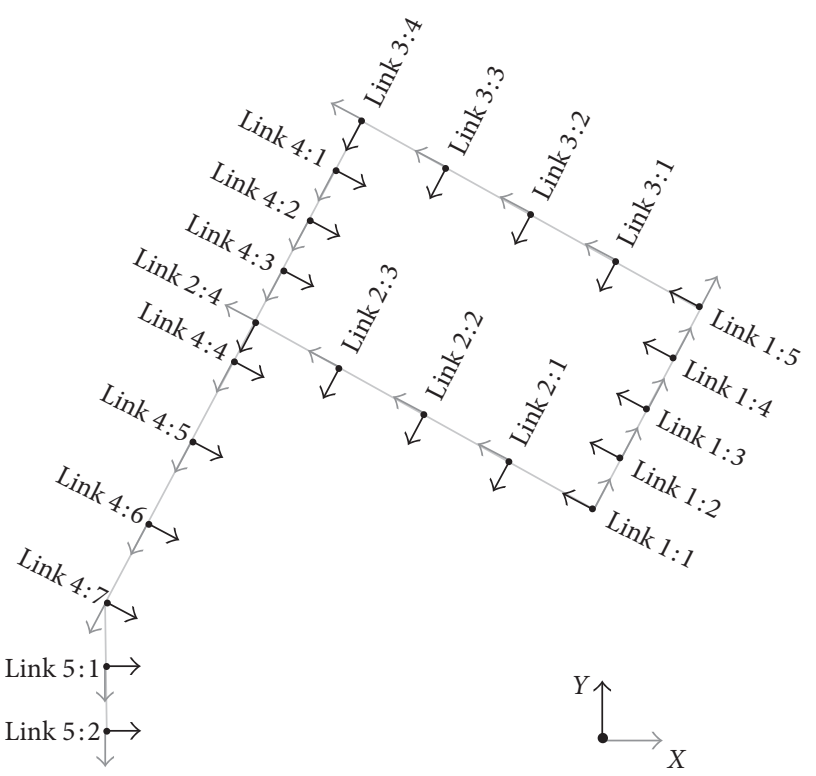

FIGURE 4: Geometry of the manipulator created in the acquisition software, LMS Test.Lab 11B.

parameters. On the contrary, uncertainty on the Young's modulus of the beam is also useful to account for the presence of joint compliance, which reduces the overall stiffness of the links. As far as the beam mass density is concerned, it has been included among the uncertain parameters since the exact value is unknown (only a nominal value reflecting the chemical composition was available) and in order to account for the presence of screws and cables. For this parameter, a unique value for all the links has been adopted. The lengths of the links and their cross-sectional areas are instead assumed as exact values.

Feasible values have been constrained by upper and lower bounds, which are listed in Table 3 together with the original and the updated values for each parameter. The original values have been taken from nominal data of the parameters (e.g., nominal value of mass density in accordance with the alloy composition). The bounds have been defined as a judicious percentage of the nominal parameters (e.g., $\pm 5 \%$ of the nominal value in the case of $\rho$ ). Additionally, a constraint on the total mass has been included, by formulating it through (24). The bounds on friction forces $\left(\mathbf{h}_{\mathrm{L}}\right.$ and $\left.\mathbf{h}_{\mathrm{U}}\right)$, for the static equilibrium constraints, have been estimated by operating the motor in a torque-controlled mode with increasing torques and by repeating the test many times (e.g., to account for uncertainty due to friction variations with temperature and for hysteresis, whose modeling is not of interest for this work).

The following notation has been employed for the subscripts in Table 3: numbers from 1 to 5 denote the links (Figure 3(b)), letters from $\mathrm{A}$ to $\mathrm{E}$ indicate the joints, $\mathrm{H}$ indicates the lumped mass of the balancing spring, and $\mathrm{F}$ indicates the one attached to link 5; the number adjacent to the letter denotes the link which each lumped mass is connected to. A total number of 29 parameters have been updated.

The modifications have been weighed in such a way that the cost of modifying the nodal inertias is lower. Indeed, their original values have been set to zero in the initial nominal model and thus are more uncertain:

$$
\lambda \boldsymbol{\Omega}=10^{5}\left[\begin{array}{cc}
\mathbf{I}_{[22 \times 22]} & \mathbf{0}_{[22 \times 7]} \\
\mathbf{0}_{[7 \times 22]} & 10^{-3} \cdot \mathbf{I}_{[7 \times 7]}
\end{array}\right] .
$$

5.5. Numerical Computation of the Jacobian Matrices. In order to speed up the implementation, numerical computation of the Jacobian matrices introduced in (9) can be exploited. The need for numerical computation is exacerbated in the case under investigation where a medium/large-scale model is updated. The great issue to be handled is the ill-conditioning of matrices of this kind of systems, which have both low-frequency and high-frequency vibrational modes. Rather than using the intuitive approach of finite differences, which imposes subtractions in the numerator that lead to dangerous cancellation errors, this work proposes the use of the complex step differentiation [33]. Such a method is based on the Taylor expansion of a complex function. Let us consider the computation of $\mathbf{J}_{m_{h}}$ (the extension for $\mathbf{J}_{k_{h}}$ is obvious) by representing $\mathbf{M}^{O}$ as a complex function (with $j=\sqrt{-1}$ ) and then look at its Taylor expansion:

$$
\mathbf{M}^{O}\left(m_{h}^{O}+j \varepsilon\right)=\mathbf{M}^{O}+\left[\frac{\partial \mathbf{M}^{O}}{\partial m_{h}^{O}}\right] j \varepsilon-\left[\frac{\partial^{2} \mathbf{M}^{O}}{\partial m_{h}^{O 2}}\right] \varepsilon^{2}+o\left(\varepsilon^{3}\right) .
$$

Hence, the computation of the Jacobian is straightforward by setting $\varepsilon$ as an extremely small real number (such as 


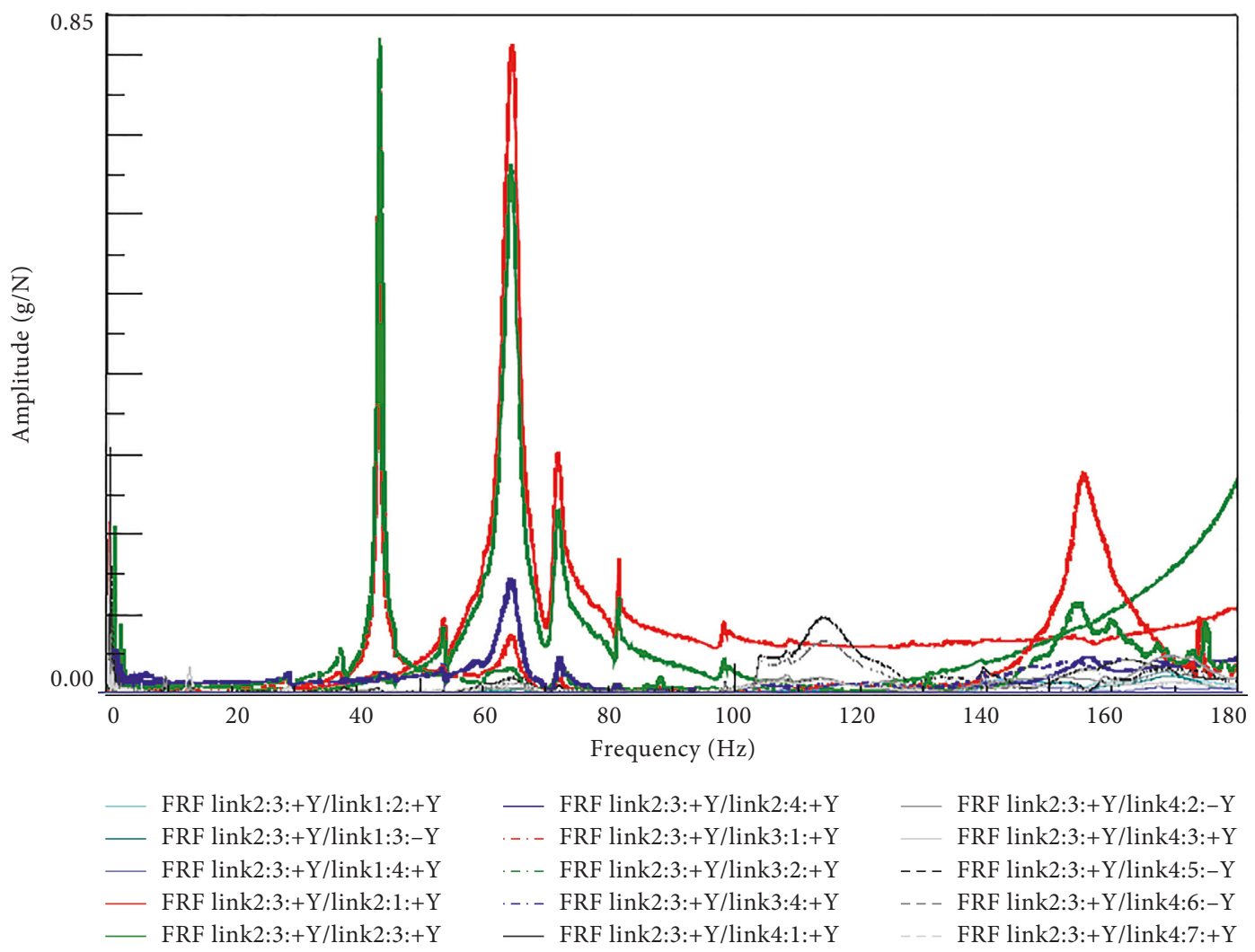

FIGURE 5: 7th row of the experimental frequency response matrix.

TABLE 3: Updating parameters.

\begin{tabular}{|c|c|c|c|c|c|}
\hline Index & Parameter & Nominal value & Lower bound & Upper bound & Updated value \\
\hline 1 & $\rho\left(\mathrm{kg} / \mathrm{m}^{3}\right)$ & 2710 & 2574.5 & 2845.5 & 2742.6 \\
\hline 2 & $E_{1}(\mathrm{~Pa})$ & $69 \cdot 10^{9}$ & $65.55 \cdot 10^{9}$ & $72.45 \cdot 10^{9}$ & $65.55 \cdot 10^{9}$ \\
\hline 3 & $E_{2}(\mathrm{~Pa})$ & $69 \cdot 10^{9}$ & $65.55 \cdot 10^{9}$ & $72.45 \cdot 10^{9}$ & $65.55 \cdot 10^{9}$ \\
\hline 4 & $E_{3}(\mathrm{~Pa})$ & $69 \cdot 10^{9}$ & $65.55 \cdot 10^{9}$ & $72.45 \cdot 10^{9}$ & $71.07 \cdot 10^{9}$ \\
\hline 5 & $E_{4}(\mathrm{~Pa})$ & $69 \cdot 10^{9}$ & $65.55 \cdot 10^{9}$ & $72.45 \cdot 10^{9}$ & $69.69 \cdot 10^{9}$ \\
\hline 6 & $E_{5}(\mathrm{~Pa})$ & $69 \cdot 10^{9}$ & $65.55 \cdot 10^{9}$ & $72.45 \cdot 10^{9}$ & $66.04 \cdot 10^{9}$ \\
\hline 7 & $k_{\mathrm{bs}}(\mathrm{N} / \mathrm{m})$ & 1520 & 1216 & 1824 & 1520 \\
\hline 8 & $M_{\mathrm{A}, 1}(\mathrm{~kg})$ & 7.644 & 6.115 & 9.172 & 7.645 \\
\hline 9 & $M_{\mathrm{B}, 1}(\mathrm{~kg})$ & 0.392 & 0.313 & 0.470 & 0.392 \\
\hline 10 & $M_{\mathrm{A}, 2}(\mathrm{~kg})$ & 9.517 & 7.613 & 11.420 & 9.523 \\
\hline 11 & $M_{\mathrm{H}, 2}(\mathrm{~kg})$ & 0.400 & 0.320 & 0.480 & 0.400 \\
\hline 12 & $M_{\mathrm{D}, 2}(\mathrm{~kg})$ & 0.648 & 0.518 & 0.777 & 0.650 \\
\hline 13 & $M_{\mathrm{B}, 3}(\mathrm{~kg})$ & 0.671 & 0.536 & 0.805 & 0.671 \\
\hline 14 & $M_{\mathrm{C}, 3}(\mathrm{~kg})$ & 0.383 & 0.306 & 0.459 & 0.383 \\
\hline 15 & $M_{\mathrm{C}, 4}(\mathrm{~kg})$ & 0.657 & 0.526 & 0.789 & 0.660 \\
\hline 16 & $M_{\mathrm{D}, 4}(\mathrm{~kg})$ & 0.308 & 0.246 & 0.369 & 0.308 \\
\hline 17 & $M_{\mathrm{E}, 4}(\mathrm{~kg})$ & 1.844 & 1.537 & 2.151 & 1.844 \\
\hline 18 & $M_{\mathrm{E}, 5}(\mathrm{~kg})$ & 0.114 & 0.095 & 0.133 & 0.114 \\
\hline 19 & $M_{\mathrm{F}, 5}(\mathrm{~kg})$ & 0.046 & 0.036 & 0.055 & 0.046 \\
\hline 20 & $J_{\mathrm{A}, 1}\left(\mathrm{~kg} \cdot \mathrm{m}^{2}\right)$ & 0.0130 & 0.0104 & 0.0156 & 0.0130 \\
\hline 21 & $J_{\mathrm{A}, 2}\left(\mathrm{~kg} \cdot \mathrm{m}^{2}\right)$ & 0.0229 & 0.0183 & 0.0275 & 0.0229 \\
\hline 22 & $J_{\mathrm{E}, 5}\left(\mathrm{~kg} \cdot \mathrm{m}^{2}\right)$ & 0.0015 & 0.0012 & 0.0018 & 0.0015 \\
\hline 23 & $J_{\mathrm{B}, 1}\left(\mathrm{~kg} \cdot \mathrm{m}^{2}\right)$ & 0 & 0 & 0.0005 & 0.0005 \\
\hline 24 & $J_{\mathrm{B}, 3}\left(\mathrm{~kg} \cdot \mathrm{m}^{2}\right)$ & 0 & 0 & 0.0005 & $4.91 \mathrm{e}-4$ \\
\hline 25 & $J_{\mathrm{C}, 3}\left(\mathrm{~kg} \cdot \mathrm{m}^{2}\right)$ & 0 & 0 & 0.0005 & $5 e-4$ \\
\hline 26 & $J_{\mathrm{C}, 4}\left(\mathrm{~kg} \cdot \mathrm{m}^{2}\right)$ & 0 & 0 & 0.0005 & $4.37 \mathrm{e}-4$ \\
\hline 27 & $J_{\mathrm{D}, 4}\left(\mathrm{~kg} \cdot \mathrm{m}^{2}\right)$ & 0 & 0 & 0.0005 & $4.03 \mathrm{e}-4$ \\
\hline 28 & $J_{\mathrm{D}, 2}\left(\mathrm{~kg} \cdot \mathrm{m}^{2}\right)$ & 0 & 0 & 0.0005 & $0.01 \mathrm{e}-4$ \\
\hline 29 & $J_{\mathrm{E}, 4}\left(\mathrm{~kg} \cdot \mathrm{m}^{2}\right)$ & 0 & 0 & 0.0012 & 0.0012 \\
\hline
\end{tabular}


TABLE 4: Comparison of the experimental and analytical modal properties.

\begin{tabular}{|c|c|c|c|c|c|c|}
\hline $\begin{array}{l}\text { Frequency } \\
\text { (experimental) } \\
(\mathrm{Hz})\end{array}$ & $\begin{array}{c}\text { Frequency } \\
\text { (nominal model) } \\
(\mathrm{Hz})\end{array}$ & $\begin{array}{c}\text { Frequency } \\
\text { (updated model) } \\
(\mathrm{Hz})\end{array}$ & $\begin{array}{l}\varepsilon_{i}(\text { nominal } \\
\text { model })(\%)\end{array}$ & $\begin{array}{l}\varepsilon_{i} \text { (updated } \\
\text { model) }(\%)\end{array}$ & $\begin{array}{c}\text { MAC } \\
\text { (nominal model) }\end{array}$ & $\begin{array}{c}\text { MAC } \\
\text { (updated model) }\end{array}$ \\
\hline 13.38 & 13.72 & 13.27 & 2.54 & -0.82 & 0.946 & 0.949 \\
\hline 43.56 & 43.89 & 43.08 & 0.76 & -1.12 & 0.986 & 0.989 \\
\hline 64.66 & 66.96 & 64.83 & 3.56 & 0.26 & 0.967 & 0.968 \\
\hline 112.99 & 127.25 & 120.77 & 12.62 & 6.89 & 0.856 & 0.872 \\
\hline 138.17 & 147.23 & 142.30 & 6.56 & 2.99 & 0.830 & 0.830 \\
\hline 154.75 & 171.95 & 156.25 & 11.11 & 0.97 & 0.930 & 0.945 \\
\hline 198.57 & 204.93 & 198.63 & 3.20 & 0.03 & 0.967 & 0.972 \\
\hline
\end{tabular}
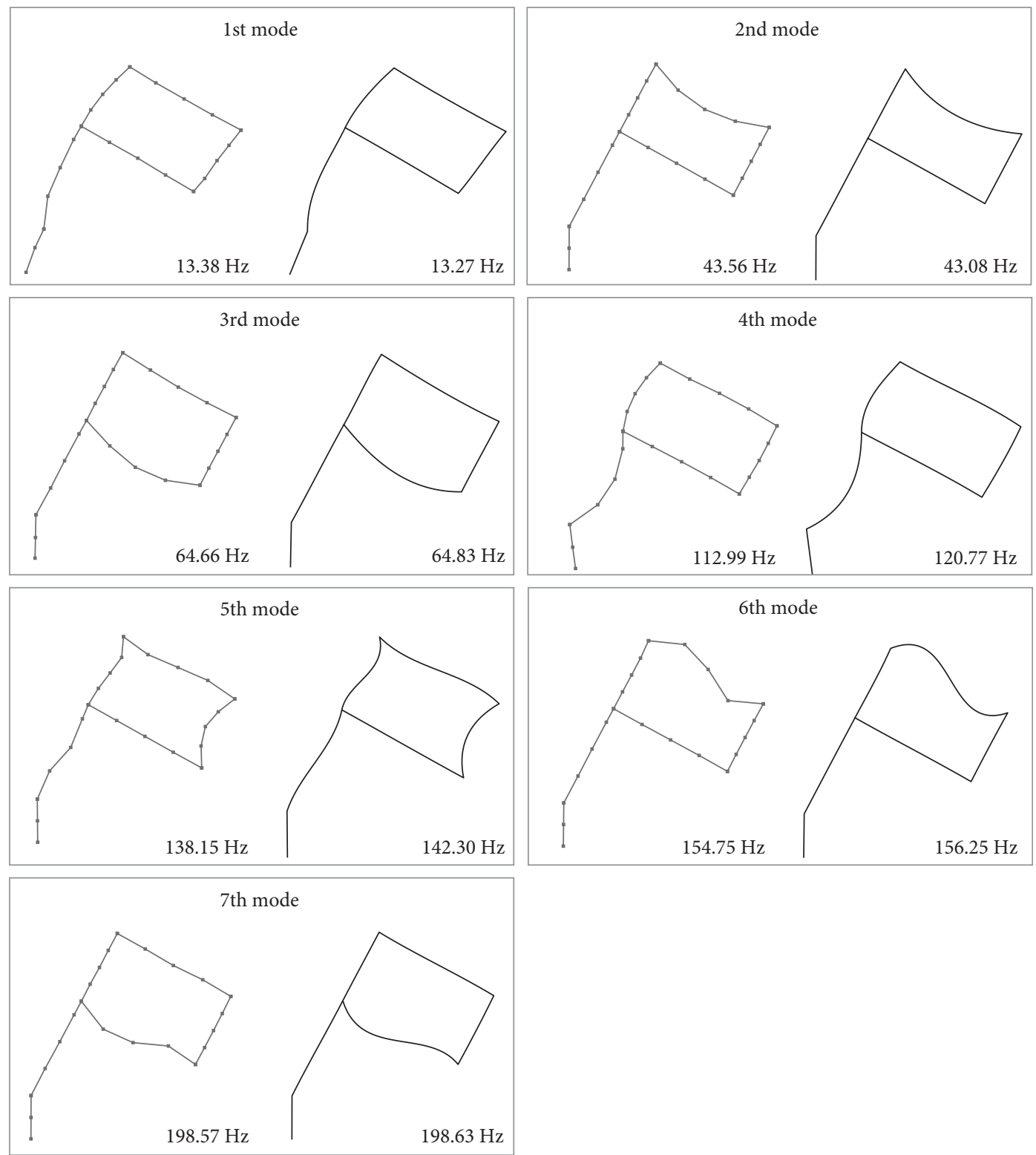

$\longrightarrow$ Experimental measurements

— Updated_model

FIGURE 6: Shapes of the seven vibrational modes: experimental (left) and updated (right) modes. 
selecting values approaching the machine precision) as follows:

$$
\mathbf{J}_{m_{j}}=\frac{\operatorname{Im}\left(\mathbf{M}^{O}\left(m_{h}^{O}+j \varepsilon\right)\right)}{\varepsilon}+o\left(\varepsilon^{2}\right) .
$$

The resulting Jacobians have negligible approximation errors, whose magnitude is close to machine precision, and require small computational effort.

5.6. Experimental Results. The effectiveness of the updating has been evaluated by computing the eigenfrequencies and the eigenvectors of the updated model and by comparing them with the experimental results by means of the percentage frequency error $\varepsilon_{i}$ :

$$
\varepsilon_{i}=100 \frac{\omega_{i}-\widehat{\omega}_{i}}{\widehat{\omega}_{i}},
$$

and the modal assurance criterion (MAC):

$$
\text { MAC }=\frac{\left(\boldsymbol{\eta}_{i}^{T} \widehat{\boldsymbol{\eta}}_{i}\right)^{2}}{\left\|\boldsymbol{\eta}_{i}\right\|^{2}\left\|\widehat{\boldsymbol{\eta}}_{i}\right\|^{2}} .
$$

The results provided by the updated model are also compared with those obtained through the original nominal model (which is the one synthesized through nominal parameters of links, like the one in [22]). In (31) and (32), $\omega_{i}$ and $\boldsymbol{\eta}_{i}$ are the $i$ th eigenfrequency and eigenvector estimated through the models $(i=1, \ldots, 7)$, while $\widehat{\omega}_{i}$ and $\widehat{\boldsymbol{\eta}}_{i}$ are the measured ones.

The comparison proposed in Table 4 shows that model updating has improved the model coherence with the experimental measurements. The greatest improvement is obtained in the estimation of the eigenfrequencies whose values are significantly missed in the nominal model. The overall absolute error (i.e., $\sum_{i=1}^{7}\left|\omega_{i}-\hat{\omega}_{i}\right|$ ) decreases from $49.85 \mathrm{~Hz}$ in the original model, to just $14.23 \mathrm{~Hz}$ after updating. Indeed, the percentage frequency error for the updated model is smaller than $1 \%$ in four modes, while the average value of such an index is equal to $1.31 \%$ (compared to the $5.76 \%$ in the original model). As for the MAC, a less relevant improvement has been obtained since the mode shape specifications are accurately met also by the original model. Nonetheless, the average MAC increases from 0.92 to 0.93 after model updating. Both these parameters are highly satisfactory also in the light of the typical correlation criteria set by military and aerospace standards which typically require $0.8-0.9$ for the $\mathrm{MAC}$ and $3-5 \%$ for the frequency error [34]. Additionally, compared with the results of preliminary studies in [22], this new formulation ensures better results in terms of both the frequency error and the MAC despite the presence of a larger number of vibrational modes that make model updating more challenging because of the larger number of requirements and of the increase of the matrix dimensions.

The correctness of the mode shapes is even more evident by Figure 6, which compares the experimental mode shapes (depicted on the left side of each subplot, as provided by the LMS software) with those computed through the updated

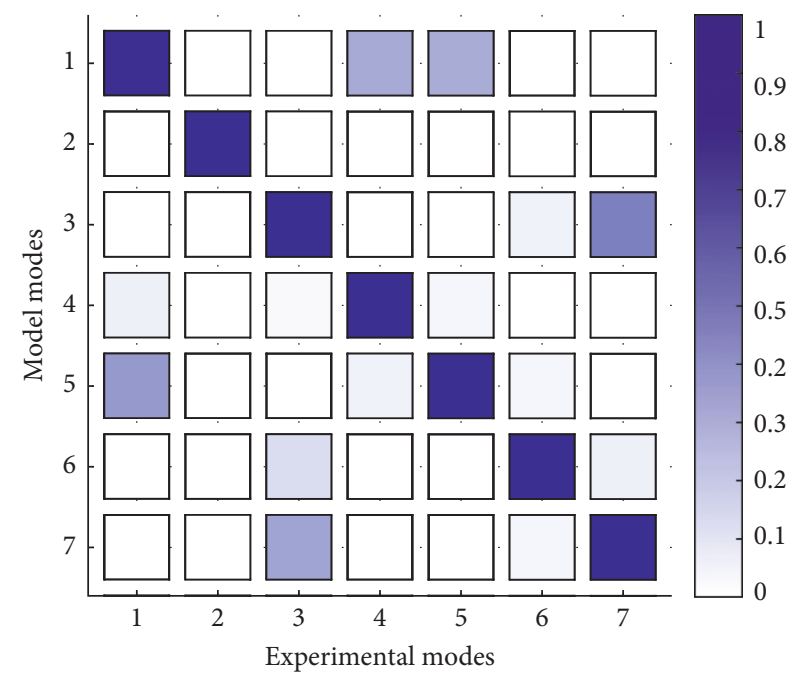

Figure 7: MAC matrix between the experimental modes and the updated model modes.

TABle 5: Comparison of the experimental and analytical modal properties.

\begin{tabular}{lcc}
\hline Frequency $(\mathrm{Hz})$ & $\begin{array}{c}\text { Modal damping } \\
\text { coefficient } \xi \\
\text { (experimental) }\end{array}$ & $\begin{array}{c}\text { Modal damping } \\
\text { coefficient } \xi \\
\text { (updated model) }\end{array}$ \\
\hline 13.39 & 0.007 & 0.007 \\
43.57 & 0.012 & 0.013 \\
64.65 & 0.017 & 0.018 \\
112.99 & 0.036 & 0.035 \\
138.15 & 0.043 & 0.040 \\
154.73 & 0.039 & 0.044 \\
198.56 & 0.048 & 0.055 \\
\hline
\end{tabular}

model (on the right). The excellent agreement is evident, thus demonstrating the correctness of the model and the effectiveness of the comprehensive approach developed in this paper.

A further proof of such a good correlation is given by Figure 7, which represents the orthogonality (defined through the canonical scalar product I) between different eigenvectors by means of the MAC matrix. Indeed, the diagonal terms are blue since they approach 1 , while the offdiagonal ones are almost zero (the average value of the offdiagonal term is 0.052).

Finally, it should be noted that the updated model does not generate spurious modes. This is a further proof of the correctness of the approach and of the results obtained.

5.7. Updating of the Rayleigh Coefficients of the Damping Model. A Rayleigh damping model (modal damping model) has been assumed since it is a popular and effective approach to represent damping [27, 28, 35, 36]. Hence, damping matrix $\mathbf{C}_{e}$ is a linear combination of mass and stiffness matrices through the Rayleigh coefficients $\alpha$ and $\beta$ :

$$
\mathbf{C}_{\mathrm{e}}=\alpha \mathrm{M}_{\mathrm{e}}+\beta \mathrm{K}_{\mathrm{e}} \text {. }
$$


The two values have been computed through leastsquares fitting of the damping of the seven modes under investigations, by taking advantage of the relation between natural frequency and modal damping of each mode:

$$
\xi_{i}=\frac{1}{2 \omega_{i}} \alpha+\frac{\omega_{i}}{2} \beta .
$$

Writing (34) for each mode leads to the following solution (" $\dagger$ " denotes the pseudoinverse matrix):

$$
\left\{\begin{array}{c}
\alpha \\
\beta
\end{array}\right\}=\left[\begin{array}{cc}
\frac{1}{2 \omega_{1}} & \frac{\omega_{1}}{2} \\
\vdots & \vdots \\
\frac{1}{2 \omega_{7}} & \frac{\omega_{7}}{2}
\end{array}\right]^{\dagger}\left\{\begin{array}{c}
\xi_{1} \\
\vdots \\
\xi_{7}
\end{array}\right\} .
$$

The following values have been identified: $\alpha=0.57$ and $\beta=8.8 e-5$, that lead to the results summarized in Table 5 . An effective estimation is therefore provided by the damping model assumed.

\section{Conclusions}

A strategy for updating models of flexible-link mechanisms has been proposed and validated in this paper. The proposed formulation leads to a constrained inverse eigenvalue problem, which makes the approach suitable for updating these complicated models. Indeed, it has the following advantages:

(i) It can handle an arbitrary number of eigenpairs and updating parameters.

(ii) Mass and stiffness matrices are simultaneously updated, to obtain more reliable results.

(iii) The experimental eigenvectors can have any arbitrary normalization.

(iv) The method handles several kinds of constraints to assure physical meaning of the updating.

(v) The formulation handles the numerical issues by providing several solutions to improve numerical conditioning and hence boosting the achievement of reliable results.

The method has been applied to a very challenging test: the model of a planar, six-bar linkage mechanism with five flexible links. The model is updated to improve the representation of the 7 lowest frequency modes, that is, those within the range from 0 to $200 \mathrm{~Hz}$ corresponding to the bandwidth of interest. The experimental results obtained are highly satisfactory, given the complexity of the system investigated, the simultaneous presence of more eigenpairs to be matched, the unavoidable uncertainty in performing accurate measurements in a mechanism (which has "rigidbody" motion), and the presence of constraints bounding the parameter updating. Overall, a meaningful improvement in the eigenvector and eigenfrequency estimation has been obtained.

\section{Data Availability}

Data in the paper are not related to other published datasets. The paper lists some relevant data of the method in the tables, and other data are available from the corresponding author upon request.

\section{Conflicts of Interest}

The authors declare that they have no conflicts of interest.

\section{Acknowledgments}

This research was funded by the University of Padova through the Progetto di Ateneo 2015 CPDA157149, "Efficient modeling of flexible link manipulators for real-time state estimation."

\section{References}

[1] L. Sheng, W. Li, Y. Wang, M. Fan, and X. Yang, "Dynamic model and vibration characteristics of planar 3-RRR parallel manipulator with flexible intermediate links considering exact boundary conditions," Shock and Vibration, vol. 2017, Article ID 1582547, 13 pages, 2017.

[2] P. Boscariol and A. Gasparetto, "Model-based trajectory planning for flexible-link mechanisms with bounded jerk," Robotics and Computer-Integrated Manufacturing, vol. 29, no. 4, pp. 90-99, 2013.

[3] Q. Zhang, X. Zhang, and J. Wei, "Experimental study of active vibration control of planar 3-RRR flexible parallel robots mechanism," Shock and Vibration, vol. 2016, Article ID 4780181, 17 pages, 2016.

[4] E. Lu, W. Li, X. Yang, M. Fan, and Y. Liu, "Modelling and composite control of single flexible manipulators with piezoelectric actuators," Shock and Vibration, vol. 2016, Article ID 2689178, 14 pages, 2016.

[5] E. Barjuei, P. Boscariol, R. Vidoni, and A. Gasparetto, "Robust control of three-dimensional compliant mechanisms," Journal Dynamic Systems, Measurements and Control, vol. 138, no. 10, article 101009, 2016.

[6] A. Abe, "Trajectory planning for residual vibration suppression of a two-link rigid-flexible manipulator considering large deformation," Mechanism and Machine Theory, vol. 44, no. 9, pp. 1627-1639, 2009.

[7] P. Kalra and A. M. Sharan, "Accurate modelling of flexible manipulators using finite element analysis," Mechanism and Machine Theory, vol. 26, no. 3, pp. 299-313, 1991.

[8] M. Giovagnoni, "A numerical and experimental analysis of a chain of flexible bodies," Journal Dynamic Systems, Measurements and Control, vol. 116, no. 1, pp. 73-80, 1994.

[9] R. Vidoni, A. Gasparetto, and M. Giovagnoni, "Design and implementation of an ERLS-based 3-D dynamic formulation for flexible-link robots," Robotics and Computer-Integrated Manufacturing, vol. 29, no. 2, pp. 273-282, 2013.

[10] J. E. Mottershead and M. I. Friswell, "Model updating in structural dynamics: a survey," Journal of Sound and Vibration, vol. 167, no. 2, pp. 347-375, 1993.

[11] S. Sehgal and H. Kumar, "Structural dynamic model updating techniques: a state of the art review," Archives of Computational Methods in Engineering, vol. 23, no. 3, pp. 515-533, 2016.

[12] J. H. Lim, D. S. Hwang, D. Sohn, and J. G. Kim, "Improving the reliability of the frequency response function through 
semi-direct finite element model updating," Aerospace Science and Technology, vol. 54, pp. 59-71, 2016.

[13] J. Jiang and Y. Yuan, "Updating stiffness and hysteretic damping matrices using measured modal data," Shock and Vibration, vol. 2018, Article ID 3727021, 7 pages, 2018.

[14] G. H. Kim and Y. S. Park, "An improved updating parameter selection method and finite element model update using multiobjective optimisation technique," Mechanical Systems and Signal Processing, vol. 18, no. 1, pp. 59-78, 2004.

[15] S. Weng, Y. Xia, Y. L. Xu, and H. P. Zhu, "Substructure based approach to finite element model updating," Computers and Structures, vol. 89, no. 8-9, pp. 772-782, 2011.

[16] Y. Yang, J. Chen, F. Lan, F. Xiong, and Z. Zeng, "Joints parameters identification in numerical modeling of structural dynamics," Shock and Vibration, vol. 2018, Article ID 2365759, 11 pages, 2018.

[17] Y. Jun, H. Ouyang, and J. F. Zhang, "A new method of updating mass and stiffness matrices simultaneously with no spillover," Journal of Vibration and Control, vol. 22, no. 5, pp. 1181-1189, 2016.

[18] M. Chouksey, J. K Dutt, and S. V. Modak, "Model updating of rotors supported on journal bearings," Mechanism and Machine Theory, vol. 71, pp. 52-63, 2014.

[19] M. Sanayei, A. Khaloo, M. Gul, and F. N. Catbas, "Automated finite element model updating of a scale bridge model using measured static and modal test data," Engineering Structures, vol. 102, pp. 66-79, 2015.

[20] E. Patelli, Y. Govers, M. Broggi, H. M. Gomes, M. Link, and J. E. Mottershead, "Sensitivity or Bayesian model updating: a comparison of techniques using the DLR AIRMOD test data," Archive of Applied Mechanics, vol. 87, no. 5, pp. 905925, 2017.

[21] I. Boulkaibet, L. Mthembu, T. Marwala, M. I. Friswell, and S. Adhikari, "Finite element model updating using Hamiltonian Monte Carlo techniques," Inverse Problems in Science and Engineering, vol. 25, no. 7, pp. 1042-1070, 2017.

[22] R. Belotti, G. Caneva, I. Palomba, D. Richiedei, and A. Trevisani, "Model updating in flexible-link multibody systems," Journal of Physics: Conference Series, vol. 744, article 012073, 2016.

[23] R. Caracciolo, D. Richiedei, and A. Trevisani, "Deformation control in rest-to-rest motion of mechanisms with flexible links," Shock and Vibration, vol. 2018, Article ID 9016028, 9 pages, 2018.

[24] R. Caracciolo, D. Richiedei, and A. Trevisani, "Robust piecewise-linear state observers for flexible link mechanisms," ASME Journal Dynamic Systems, Measurements and Control, vol. 130, no. 3, article 031011, 2008.

[25] U. Lugris, M. A. Naya, F. Gonzalez, and J. Cuadrado, "Performance and application criteria of two fast formulations for flexible multibody dynamics," Mechanics Based Design of Structures and Machines, vol. 35, pp. 381-404, 2007.

[26] F. González, P. Masarati, J. Cuadrado, and M. A. Naya, "Assessment of linearization approaches for multibody dynamics formulations," Journal of Computational and Nonlinear Dynamics, vol. 12, no. 4, article 041009, 2016.

[27] M. Liu and D. G. Gorman, "Formulation of Rayleigh damping and its extensions," Computers and Structures, vol. 57, no. 2, pp. 277-285, 1995.

[28] I. Chowdhury and S. P. Dasgupta, "Computation of Rayleigh damping coefficients for large systems," International Journal of Space Structures, vol. 43, no. 22-23, pp. 6855-6868, 2003.

[29] Z. X. Yuan and K. P. Yu, "Finite element model updating of damped structures using vibration test data under base excitation," Journal of Sound and Vibration, vol. 340, pp. 303-316, 2015.

[30] D. Calvetti, L. Reichel, and A. Shuibi, "L-curve and curvature bounds for Tikhonov regularization," Numerical Algorithms, vol. 35, no. 2-4, pp. 301-314, 2004.

[31] B. Peeters, G. Lowet, H. Van der Auweraer, and J. Leuridan, "A new procedure for modal parameter estimation," Sound and Vibration, vol. 38, pp. 24-29, 2004.

[32] A. S. Nobari and D. J. Ewins, "On the effectiveness of using only eigenvalues in structural model updating problems," Shock and Vibration, vol. 1, no. 4, pp. 339-348, 1994.

[33] J. R. Martins, P. Sturdza, and J. J. Alonso, "The complex-step derivative approximation," ACM Transactions on Mathematical Software, vol. 29, no. 3, pp. 245-262, 2003.

[34] European Cooperation for Space Standardization, Space Engineering: Modal Survey Assessment, ECSS-E-ST-32-11C, European Cooperation for Space Standardization, Noordwijk, Netherlands, 2008.

[35] X. Wang, K. Liu, H. Liu, and Y. He, "Damping Identification with Acceleration Measurements Based on Sensitivity Enhancement Method," Shock and Vibration, vol. 2018, Article ID 6476783, 16 pages, 2018.

[36] F. Asma and A. Bouazzouni, "Finite element model updating using FRF measurements," Shock and Vibration, vol. 12, no. 5, pp. 377-388, 2005. 


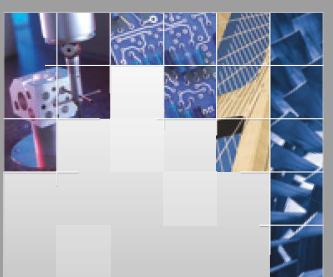

\section{Enfincering}
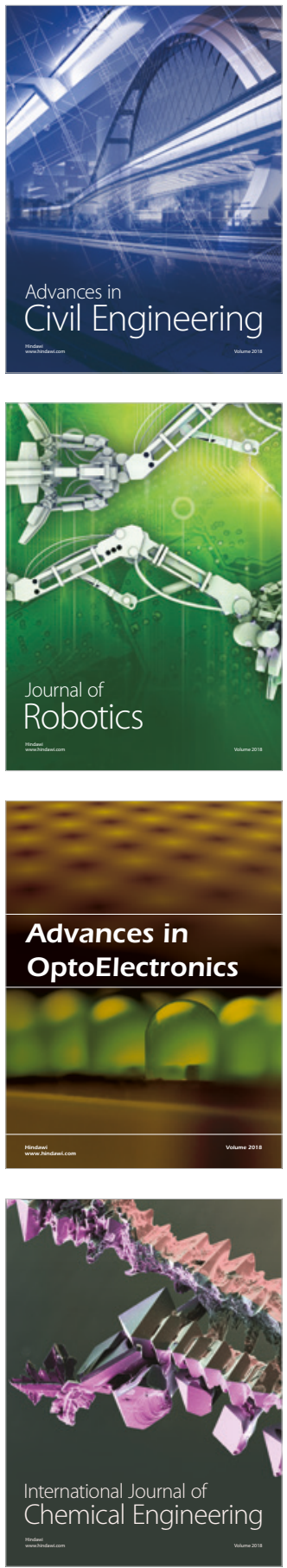

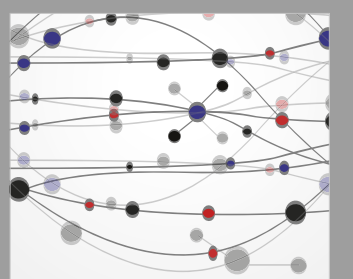

\section{Rotating \\ Machinery}

The Scientific World Journal

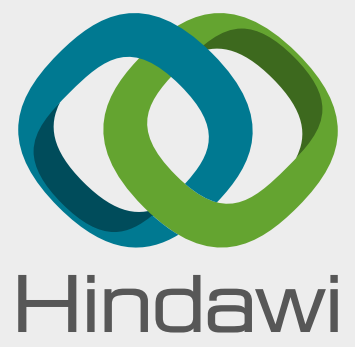

Submit your manuscripts at

www.hindawi.com
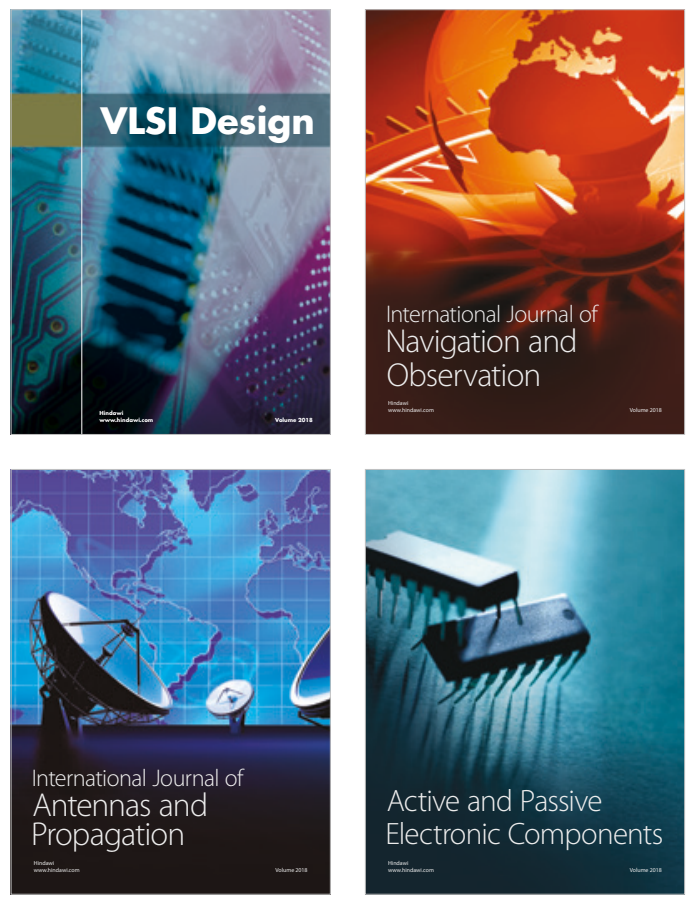
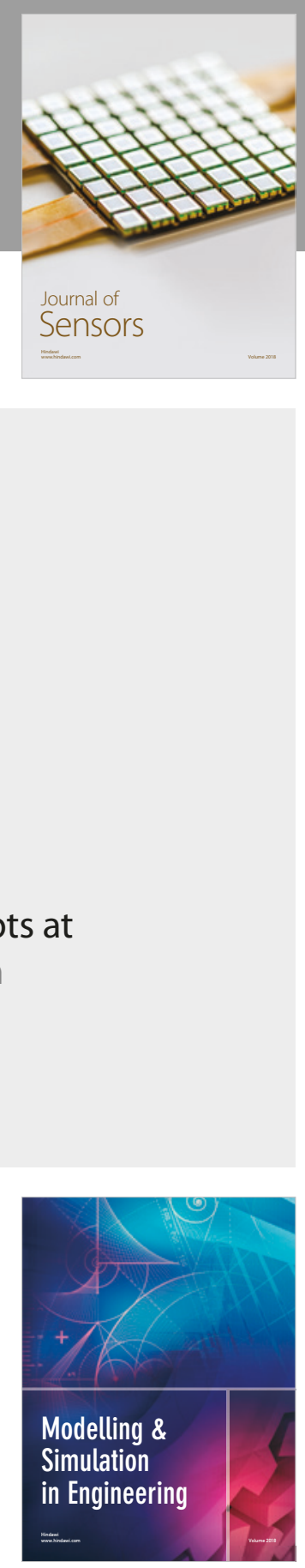

\section{Advances \\ Multimedia}
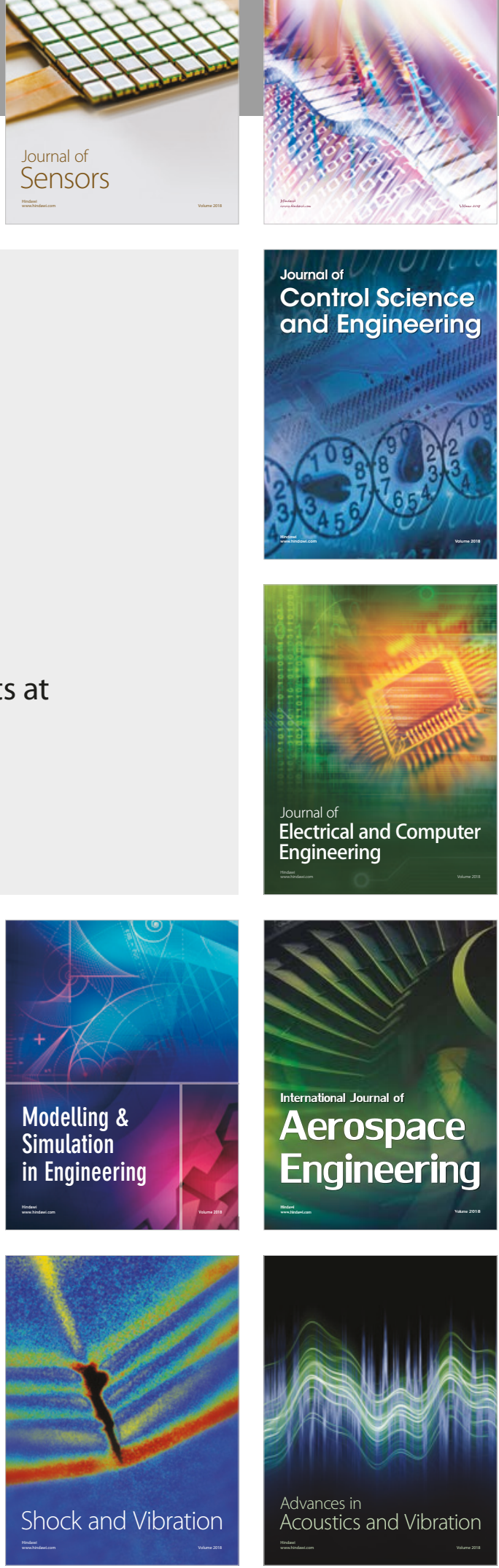